\title{
OPTICAL INVESTIGATIONS IN MOLECULAR AND IN CELL STRUCTURE
}

\begin{abstract}
$\mathrm{T}$ HE Discussions of the Faraday Society are well known all over the world: and they regularly attract distinguished visitors. The latest in the series of these Discussions was held during September 2528 at Cambridge, and visitors from abroad accounted for nearly a hundred of the three hundred present. Almost every Western European country was represented, and a large group from the United States gave a most interesting international aspect to the whole affair. The first two days of the conference were devoted to a discussion of spectroscopy and molecular structure, the remaining day and a half being concerned with the applications of various spectroscopic and microscopic techniques to the study of cell structure.
\end{abstract}

\section{Spectroscopy and Molecular Structure}

Spectroscopy is so vast a topic that it can only be dealt with in sections. The most natural beginning - which was actually adopted-is with electronic spectra. In this field it was most interesting to see the changes that have taken place since this subject was last discussed by the Faraday Society. In the first place, all but one of the papers were concerned with relatively large molecules, such as naphthalene or pyridine. This expresses a shift of emphasis away from the well-worked diatomic molecules to that border region where possible relations are being sought between molecular properties and biological activity. It was really most impressive-and encouraging - to see that at last it is becoming possible to characterize the various bands that are observed. Theory plays its part by means of group theory, enabling decisions to be made concerning the symmetries of the allowed wave functions and the permitted transitions. But it goes even further, since many of the most interesting molecular spectra arise from what are technically forbidden transitions. Such transitions, which are usually relatively weak, are made possible by the presence of certain types of vibration, and by certain spin-interactions. The various possibilities can all be listed, so that now, for the first time, we really believe that we can assign some of the transitions in naphthalene and other members of the homolc gous series. The experimental work of Dr. M. Kasha, in the United States, was the outstanding paper in this section. His analysis of singlet-triplet transitions in substituted benzenes and naphthalenes, in which he has studied the way in which an increase in atomic number of the substituent increases the intensity of these forbidden transitions by breaking down the usual form of spin coupling, and introducing a spin-orbit interaction, will probably become classic.

In the second place, it was interesting to see the greater emphasis, also expressed in the contributions dealing with the infra-red and Raman effect, on intensities. Here is a much-neglected tool, the proper use of which is most powerful.

Two other points in electronic spectra deserve mention. The first is that we seem to have passed out of the stage in which exponents of one or other type of theoretical approximation (molecular-orbital or valence-bond) fought hard with the exponents of the other approximation. In certain cases a reconciliation has been effected, with concordant predictions; and in others, we understand the natural liabilities of the methods so much better that we now adopt an attitude of modesty when expressing our conclusions. Quite clearly, theory can help enormously; but it can never dispense with experiment. Secondly, thanks largely to the work of W. C. Price of King's College, London, we are getting on better terms with the so-called Rydberg levels in molecules; here an electron is excited to an orbit of relatively large dimensions, generally lying mostly outside the greater part of the molecule. The energies of these orbits fit into a Rydberg series, very similar to those found with isolated atoms. The quantum defect in the Rydberg expression gives indications of the $s, p$, ... type of the transition, and the constant term gives valuable information about the ionization potential. Excellent progress is being made in understanding both of these, and in correlating them with the size and structure of the molecule.

The conference next turned to discuss the vibrational spectra of small molecules. Here the emphasis is quite different. Very little interest is now attached to the process of assigning frequencies and choosing force-fields; for the tools with which we study infrared spectra are now fully grown-up. The modern double-beam self-recording spectrometers do in less than a minute what used to require about two weeks ; and recent dispersing prisms have greatly extended the range of measurement, while new photocells have completely changed the precision of recording in regions up to $35 \mu$. As a result the technical problems are almost all solved; what is happening is a gradual transference of interest away from chemistry into physics and biology. In its physical aspects the detailed study of intensities is giving information about the variation of dipole moment with bonddistance, about the changes in configuration that take place on crystallization or other change of state, upon order-disorder phenomena and similar physical problems. In its biological aspects it is enabling us to follow chemical reactions taking place in many kinds of biological material. The most outstanding new applications are in low temperature and in the use of polarized infra-red radiation. Some of these will be referred to later.

It was inevitable that some consideration should be given to the competing claims of microwave analysis and more standard infra-red and Raman rotation-vibration methods for investigating molecular structure. Such a comparison was much stimulated by an excellent review of recent centimetre-wave work by E. B. Wilson. In certain recent microwave work bond distances have been given in angström units to four decimal places. This suggests that there might be some chance of the older and more conventional techniques being abandoned. But as Dr. G. Herzberg (National Research Council, Ottawa) pointed out, there is a quite astonishing agreement, usually to 1 part in $10^{4}$ or $10^{5}$, between moments of inertia determined by the two methods. This conclusion, which does not appear to have been sufficiently recognized hitherto, is very reassuring. 
It is true that microwave measurements are often made with an accuracy about a hundred times greater than the infra-red and Raman measurements. But they are made on only one rotational transition (and even then, only on relatively simple molecules), and do not, by themselves, provide sufficient information to correct for anharmonicity of the vibrational motion. As a result, they do not allow us to pass from $B_{0}$ and $D_{0}$ (constants of the vibrating molecule) to $B_{e}$ and $D_{e}$ (constants of the non-vibrating molecule). At present, also, microwaves can only be used with unsymmetrical molecules. It was with something approaching relief that the infra-red spectroscopists heard all this, and very happily came to the conclusion that their subject was not yet outmoded. To me it seems probable that a combination of the older and newer techniques will prove most fruitful, in which the wider applicability of the one approach is supplemented, at suitable points, by the intrinsically more accurate, though less versatile, character of the other. The agreements already reached are a most welcome augury in this direction.

Nevertheless, the infra-red exponents had no cause for dismay - at this very meeting they described how there was an almost completely unhindered rotation in zinc and mercury dimethyl; they gave, for the first time, a molecular shape and size to the HNCO molecule, they showed that in $\mathrm{AsH}_{3}$ and $\mathrm{PH}_{3}$ there appears to be no measurable inversion spectrum such as is found in $\mathrm{NH}_{3}$; they found evidence for two related, but dissimilar, forms of the nitrate ion $\mathrm{NO}_{3}{ }^{-}$ in metallic crystals, and they discussed the interactions between lattice vibrations and internal vibrations in molecular crystals. This is substantial progress, showing that the early technical improvements of Dr. H. W. Thompson and Prof. G. B. B. M. Sutherland are continuing to bear fruit.

Yet the outstanding paper in this section was due to Prof. R. Mecke, of Freiburg-im-Breisgau, who reported briefly on a series of about a dozen papers published from his Institute in recent years. All were concerned with the 'hydrogen bond' resulting from association of alcohols. What was most impressive about these papers was the breadth of the attack made to converge on this one problem. Thus dielectric constant measurement, conductivity measurement (showing a small, but quite definite and measurable, proportion of ions due to the migration of the proton from one oxygen atom to another), absolute intensities of a series of overtones of the fundamental frequency of vibration, ultra-violet absorption, and thermal effects : all these were made to provide a picture of some of the characteristics of the $\mathrm{O}-\mathrm{H}$... O bond which could not possibly have been obtained by one technique alone. This interplay of various techniques was a valuable and stimulating warning against a too narrow outlook. One interesting by-product of this work was that Mecke was able to derive the famous Morse potential function for molecular vibrations. This was previously entirely empirical.

It is more difficult to comment on the last section of this part of the conference, which was concerned with the vibrational spectra of complex molecules. Quite clearly no one is going to try assigning force fields, so that the part played by theoretical argument is very small. Much of the work is in the nature of diagnosis, using known frequency charts to infer the existence of certain types of bonding. Intensity studies are able not only to show which types of bond occur, but also how many of them there are. Thus in saturated hydrocarbons we can estimate the ratio of the number of tertiary and primary carbons ; and in unsaturated hydrocarbons the number of double bonds. We can also follow the migration of the double bonds that accompany the chlorination of rubber. It is a great pity that the whole basis of this work-the constancy of vibration frequency of individual bonds, or groups of bonds-is still not adequately understood theoretically. But some preliminary work has already shown signs of success in sorting out the 'skeletal' vibrations of a long-chain molecule from the 'rocking' and 'wagging' frequencies of individual units along the chain.

As an empirical tool, the most noteworthy recent applications of infra-red investigations to large molecules are in the use of polarized radiation; for by controlling the direction of polarization relative to the crystal, we can trace the directions in which, for example, most of the $\mathrm{N}-\mathrm{H}$. . . O bonds of a protein system are oriented. We can distinguish between folded and extended protein chains, and the process of crystallization can be followed in a most effective manner. The significance of this technique as a tool for investigating crystal structure is that the absorption of radiation is highly specific to certain bonds, and in this way we have a kind of 'probe' that can penetrate easily into the system, by-passing groups of atoms in which we are not interested, and telling us about the orientation of bonds that would otherwise be inaccessible. It may be true that there is still need for caution in interpreting the observations, but there is no doubt whatever that an enormous field of application of this type of study to systems of biological interest lies open to be exploited.

The Discussions of the Faraday Society have a habit of marking turning-points in their subject. Very few people would deny that this was true in the. field of electronic and vibrational spectra, as revealed in the recent Discussion at Cambridge.

\section{A. Coulson}

\section{Optical Methods of Investigating Cell Structure}

The second part of the Faraday Society Discussion was an intentional departure in subject from anything the Society has previously attempted, and marks a direct recognition and encouragement by the Society of biophysiral research. In 1929 a Colloid Committee was formed and has been of great value in stimulating and suggesting Discussions. This Committee has recently been reconstituted as the Colloid and Biophysics Committee, and has already been responsible for the important meeting to discuss lipoproteins, held at Birmingham last year, together with a number of other locally organised discussions, such as those on nucleic acids, water transport in plants, and the electrical double layer.

The Faraday Society, which, since its foundation in 1909, has had such an important influence on the development of the common ground between physics and chemistry, now recognizes at a critical period the borderline subjects associated with physics, chemistry and biology; and it is the intention of the Society to encourage publication in this field. In reviewing the Cambridge meeting, I shall not attempt a detailed report of all items of discussion, but rather to give the background and set out briefly the main features of importance which arose.

The microscope has always, for obvious reasons, been a fundamental physical tool in cytology. During 


\section{No. 4228 November 11, $1950 \quad$ N A T U R E}

the past twenty years the development of phasecontrast microscopy, the reflecting microscope-and in a wider field the electron microscope-have led to now attempts to understand in a more fundamental way the structure and function of the cell. The value of the spectroscope in biology has long been recog. nized, but attempts to combine microscopes and spectrographs satisfactorily are comparatively new. Since 1936, Caspersson and his colleagues in Stockholm have made use of the long-known ultra-violet absorption of the purine and pyrimidine components of the nucleic acids in a detailed study of cells. The physical assumptions underlying the accurate appreciation of such methods have not been very well understood, and the time seemed peculiarly appropriate for the discussion under review.

Seventeen papers were presented at the meeting; broadly speaking, these fell into three groups. Apart from the introduction by the writer, which ranged over the implications of the discussion as a whole, the first group of papers was largely concerned with instruments, their design and proper use in the study of biological material.

The design of reflecting microscopes and the performance of these instruments came in for a good deal of lively discussion, started by R. Barer (University Museum, Oxford), in which K. W. Norris and M. H. F. Wilkins, of King's College, London (as authors of papers), and Keohane and Wilfred Taylor took part. Two types of reflecting microscope have been developed in Britain. The pioneer work on aspherized reflecting objectives has been done by Burch, and it is understood that some of these with 0.65 numerical aperture and 14 per cent central obstruction will be available before long. The difficulty of making aspherized surfaces on a large scale probably means, however, that such instruments will always be expensive. The other design of reflecting microscope in Britain, using simple spherical surfaces, is due to Wilkins, Norris and W. E. U. Seeds at King's College, London. An instrument with a numerical aperture of 0.65 and 35 per cent central obstruction is already commercially available. A third design, due to Grey in the United States, employs mirrors and lenses and is said to have a numerical aperture of 0.72 and obstruction ratio of $\sim 30$ per cent.

The chief point of discussion arose over the appropriate measurement of coma in the simple spherical surface objectives. Unpublished calculations by Grey (presented to the meeting by Barer) indicated that the coma-free field would be small. The experimental approach of Wilkins and collaborators utilizing a microscope interferometer test, together with study of pinhole diffraction images, appeared to show that a wider field is coma-free and entirely adequate for microspectrometric studies on cells and their components.

The discussion on these matters was largely concerned with the question as to whether the reported computations of Grey are equivalent to the stringent experimental tests applied by Wilkins and Norris to their own objectives. Coma was defined by Wilkins and Norris as just allowable at any point in the field where the intensity of the second diffraction ring in the image of a point object is equal to the intensity of the first ring on the other side of the maximum. Whether such a definition of effective field-size falls within the arbitrary criterion of the Rayleigh limit, presumably only the wave theory of aberrations can show ; no doubt this point will be cleared up at a later date. The further question as to whether an aspherized Burch objective is suitable for such purposes depends on the allowable degree of 'cobbling' discussed by Wilkins in his paper, and by Keohane at the meeting. A number of calculations by Wilkins on the size of object, and other problems, in relation to accurate absorption measurements, were also presented.

Infra-red microspectrometry has been attempted on a number of biological objects during the past two years, but the resolving power revealed in the work so far published has been low. The interest of the paper by Fraser, in which a 0.8 numerical aperture reflecting microscope was successfully combined with a Grubb Parsons infra-red spectrometer, rested on the very high spectral resolution obtained, which ranged from $8 \mathrm{~cm}^{-1}$ at $3,000 \mathrm{~cm} .^{-1}$ to $2.0 \mathrm{~cm}^{-1}$ at $1,000 \mathrm{~cm} .^{-1}$. Kletz also reported the design by Bosanquet of a reflecting objective of similar aperture for use in the infra-red.

A reminder that macrospectrometric measurements are still of great importance was given in the paper by G. H. Beaven and E. R. Holiday (London Hospital), and by E. M. Jope (Queen's University, Belfast), on ultra-violet absorption spectra of aromatic amino-acids in proteins and related compounds. The absorption spectra obtained by the logarithmic cam technique were extremely fine.

The use of microspectrographic methods in the study of crystals of biological interest was also aptly illustrated in the second group of papers presented by Seeds and Wilkins (tobacco mosaic virus, tyrosine), and M. Perutz (Cambridge), E. M. Jope and R. Barer (horse methæmoglobin); polarized ultraviolet light was used in all these investigations, and Seeds also described the design of a low-temperature stage for the reflecting microscope.

A. Engström (Institute for Cell Research, Stockholm) described work on the investigation of a number of tissues by his now well-known and elegant $\mathrm{X}$-ray absorption technique, which allows of the accurate estimation of sulphur, phosphorus and calcium. Work on the much more difficult problem of estimating carbon, oxygen and nitrogen is in progress. Thorell gave an account of a single-beam recording microspectrograph, working in the short wave-length visible range, for the study of end cellular hæmoglobin synthesis. H. Hyden (Göteborg) presented studies on human nerve cells in relation to the yellow pigment in the cytoplasm, which is at present thought to be a pterin.

These papers, and the remaining ones by $\mathrm{H}$. G. Davies and P. M. B. Walker (King's College, London), B. Commoner (Washington University), Catchpole (University of Chicago), and Bradfield (University of Cambridge); brought out several features of interest. The enthusiastic application of microspectrometric methods which has taken place since the War has perhaps led to the view that such methods could give, unaided, unequivocal answers to many fundamental questions, such as those involved in the relations of nucleic acids to protein synthesis. Such problems are essentially dynamic in nature, and it is unlikely that solutions will be obtained by static methods alone. It was refreshing to find all workers thinking very critically concerning the choice of biological material likely to lead to the most valuable results. On one hand, there is the clear-cut geometry of the epidermal hairs of plants such as Coleus, discussed in the elegant work of Commoner ; on the other, sections of pathological tissue. Intermediate between these 
extremes are tissue-culture preparations such as those used by Davies and Walker. The paper by Davies brought out very clearly the problems of ultra-violet dosage of living cells, and a film shown by Walker demonstrated the effects of continued dosage on the nucleus and cytoplasm. So far as one can judge at the present time, there is just as much danger in the indiscriminate use of qualitative ultra-violet photography as there is in over-stressing the quantitative determination of metabolites by microspectrometric methods. The effects of fixatives on the biological material are as yet only rather vaguely apprehended, and it was interesting to note the measurements of Engström using the X-ray absorption method, which demonstreited in certain instances that the use of formalin caused a decrease in mass of tissue, while the use of Carnoy led to an increase. Freeze-drying left the tissue unchanged in this respect.

The ripples which have spread from the pioneer work of C'aspersson have now affected many groups in all parts of the world, and perhaps the most significant result of the Discussion lies in the healthy critical attitude adopted by all to the use of microspectrometry in biology. The results of this will, of course, only become apparent in the future. On the other hand, it is clear that the present time is bringing forward new and improved optical methods for the study of microscopic objects, biological or otherwise, and important results are already being obtained on virus nucleoprotein and crystals of biological relevance. The discussions also made it clear that fundamental progress in all these fields can best arise from the close collaboration of physicists, chemists and biologists, in the focusing of many methods and techniques on the problems which arise.

\section{J. T. RANDALI}

\section{POPULATION STUDIES OF GREAT BRITAIN}

$\mathrm{V}$ OLUME 2 of "Papers of the Royal Commission on Population"* comprises more than a dozen reports and papers of the Statistics Committee. Although all deal with related topics each is largely self-contained, so the publication is not intended to be a unified contribution to demography.

In studying the occurrence of events in a population by statistical methods, a fundamental principle must be observed if the most efficient use is to be made of the data. The principle is, in brief, that as much concomitant information shall be available about individual members of the population who do not experience the events as about those who do. This principle is often met in practice by choosing a suitable random sample of unaffected individuals for study as a control group. In demography, however, the procedure is to relate the numbers of persons experiencing the events to the appropriate population among whom they arise. In either case, any concomitant information which is available for those experiencing the events, but not for members of the control group (or for the whole population), is of limited statistical value.

The lack of a census for Great Britain in 1941 meant that the extensive information on fertility,

- Papers of the Royal Commission on Population. Vol. 2. Reports and Selected Papers of the Statistics Committee. Pp. viii +422 . (London: H.M. Stationery Office, 1950.) 8s. net. obtained since July 1938 at birth and death registration, could not be related to equally comprehensive population statistics. The difficulty was partly met by unpublished population estimates by age, marital status and duration of marriage, which were supplied to the Statistics Committee by the General Register Office; but even so, the analysis of fertility statistics could not be as complete as was desirable, and those analyses which were made must have a considerable margin of error. It might have been expected that one of the first uses of the 1946 Family Census would have been to remedy these deficiencies, at least for the female population. It is therefore most puzzling that no such use of the Census material is even considered in the present volume, although, apparently, the necessary basic tabulation was made. In particular, it is very regrettable that, two years after the Census was taken, the research assistant of the Statistics Committee, Mr. J. Hajnal, had to embark upon an extensive study of reproductivity with only the Registrar General's approximate, and incomplete, population estimates. This inevitably restricts the scope of Mr. Hajnal's important contribution to demographic methodology.

Despite these imperfections, the fertility analyses do demonstrate beyond doubt the phenomena of 'postponement' of births and the subsequent 'making-up' which are characteristic of marriages in the period 1940-47, as compared with 1938 and 1939. The beginnings of the process can be discerned for first and second births by the end of 1943. It is shown, too, that the total number of legitimate births in the years 1939-47 was similar to the number which would have been expected if the 1938 figure of 1.93 births per marriage had persisted throughout those years. In other words, the made-up births approximately equalled those postponed. The discovery of this pattern underlying the fluctuations in birth- and marriage-rates during the War years is of considerable demographic and sociological interest.

The Family Census, which was taken in JanuaryFebruary 1946, was based upon a 10 per cent sample of women throughout Great Britain who were or had been married. Although no compulsion could be exercised, at least 87 per cent of the women in the sample responded. Various indirect tests showed that an unduly high proportion of those who did not respond must have been childless, and the results have been suitably adjusted. The preliminary report contained in the present volume gives a clear account of the practical aspects of the Census procedure, from the necessary organisation to the methods of adjustment and analysis. It also presents provisional results concerned with 'family size' (which means here the average number of live births per woman after a certain duration of marriage). It is not made sufficiently clear that these figures refer (naturally) only to those women who have survived to the Census date. There is good reason to suppose that mortality (and perhaps migration) is correlated with fertility-certain forms of cancer are well-known examples-and this may introduce a bias in the absolute figures which could be substantial for the earlier cohorts of marriages. However, the trend shown by the Census results is unlikely to be seriously misleading; and this brings out a second main point of demographic interest, namely, the recent stabilization of family size in Great Britain after a decline lasting for more than half a century. Despite the violent war-time disturbances in birth- and marriage- 